\title{
POTENCIAL ANTI-INFLAMATÓRIO E ANTIOXIDANTE DA Curcuma longa L
}

\section{ARTIGO DE REVISÃO}

CORRÊA, Josiane Santos ${ }^{1}$, FERNANDES, Gabriela Rocha², BATISTA, Maely Oliveira ${ }^{3}$, RAMOS, Denny Vitor Barbosa ${ }^{4}$, OLIVEIRA, Daniel Batasini de ${ }^{5}$, OLIVEIRA, César Augusto Batasini de ${ }^{6}$

CORRÊA, Josiane Santos. Et al. Potencial anti-inflamatório e antioxidante da Curcuma longa L. Revista Científica Multidisciplinar Núcleo do Conhecimento. Ano 06, Ed. 06, Vol. 04, pp. 87-107. Junho de 2021. ISSN: 2448-0959, Link de acesso: https://www.nucleodoconhecimento.com.br/saude/curcuma-longa, $\quad$ DOI: 10.32749/nucleodoconhecimento.com.br/saude/curcuma-longa

\section{RESUMO}

A presente pesquisa apresentou por objetivo a realização de uma pesquisa acerca da atividade antioxidante e anti-inflamatória apresentada pela espécie vegetal Curcuma longa Linn e foi desenvolvida através de levantamento bibliográfico com abordagem qualitativa de natureza descritiva, seguindo a metodologia descrita por Augusto et al. (2013). Os artigos foram selecionados nos bancos de dados DrugBank, Google acadêmico e Scielo. Os descritores de busca foram: Curcuma longa, funções biológicas, compostos bioativos, ação antioxidante, aplicação clínica da $C$. longa, ação anti-inflamatória e neutralização de radicais livres publicados no período de 2010 a 2020 nos idiomas inglês e português. A atividade anti-inflamatória da $C$. longa L. deriva de sua capacidade em inibir a produção de proteínas que

\footnotetext{
${ }^{1}$ Farmacêutica.

${ }^{2}$ Farmacêutica.

3 Pós-graduação Latu sensu em Docência do Ensino Superior, Graduação em Farmácia Generalista e Acadêmica do curso de Licenciatura em Química.

${ }^{4}$ Mestrado em Educação, Graduação em Licenciatura em Química.

${ }^{5}$ Acadêmico do curso de Farmácia.

${ }^{6}$ Orientador. Pós-graduação em Atenção Farmacêutica e Farmácia Clínica, Graduação em Farmácia Generalista.
}

RC: 87968

Disponível em: https://www.nucleodoconhecimento.com.br/saude/curcuma-longa 
fazem parte do processo inflamatório, atuando na supressão das cascatas sinalizadoras inflamatórias. Também sugere que o mecanismo pelo qual a cúrcuma expressa seu efeito é impedindo a produção da ciclooxigenase-2 (COX-2) além de inibir a expressão de interleucinas e fator de necrose tumoral- $\alpha$ (TNF- $\alpha$ ) e ser capaz de intervir na via do ácido araquidônico e interrupção da atividade do fator nuclear $\mathrm{k}$ B (NF)-KB. O óleo essencial dessa espécie apresenta atividade antioxidante devido à sinergia dos seus componentes dose-dependente sugerindo que o óleo expressa sua ação antioxidante devido a atração da doação de hidrogênio. Os extratos obtidos por extração ultrassônica manifestaram maior capacidade redutora e capacidade superior de eliminação dos radicais DPPH e ABTS. Além disso, os resultados também demonstraram que in vitro a $C$. longa L. apresenta potencial anticâncer considerável ao reduzir a produção de espécies reativas de oxigênio (EROs) intracelular, inibição da migração celular, indução de alterações morfológicas nas células e em material genético. Foi possível elucidar que a Curcuma longa L. apresenta capacidade anti-inflamatória e antioxidante comprovada in vitro, porém, ainda é perceptível a pouca demanda quando se trata de estudos in vivo principalmente em humanos. Também é possível comprovar a escassez de estudos brasileiros.

Palavras-chave: Curcuma longa L, Atividade antioxidante, Atividade anti-inflamatória.

\section{INTRODUÇÃO}

Historicamente, as plantas medicinais são utilizadas pela medicina para o tratamento de enfermidades diversas, entretanto, ao longo dos anos sua finalidade foi ampliada tanto para profilaxia quanto para o tratamento de doenças clínicas em todo o mundo, especialmente no Brasil, devido a vasta biodiversidade encontrada no país (MORETES; GERON, 2019; GRASSO, 2017; BRASIL, 2016). Nesse sentido, a sua utilização de forma empírica difundiu-se e impulsionou o crescimento de pesquisas científicas de tal maneira que o Ministério da Saúde criou, em fevereiro de 2009, a Relação Nacional de Plantas Medicinais de Interesse ao Sistema Único de Saúde 
(RENISUS). Dentre as mais variadas espécies inseridas nessa relação, encontra-se a Curcuma longa L. (BRASIL, 2009).

A C. longa L., de origem asiática, se caracteriza como uma planta herbácea pertencente à família Zingiberaceae que possui aplicação universal como tempero culinário sob a forma de pó (MARCHI, 2016; PRASAD; AGGARWAL, 2011). A espécie foi trazida ao Brasil durante o período colonial e adaptou-se facilmente ao solo brasileiro de modo que é cultivada em diversas regiões do país variando o nome de acordo com seu local de cultivo, como açafrão-da-terra, cúrcuma, turmérico, açafrão, gengibre-amarelo, raiz do sol, entre outros (MARCHI, 2016). Em relação ao seu potencial terapêutico, na medicina tradicional chinesa, a cúrcuma carrega um histórico de uso no tratamento de condições inflamatórias em todo o sudeste da Ásia além das funções antioxidante, antimicrobiana, antitumoral, anticancerígena, antifúngica, antimalárica, anticoagulante, antiespasmódica, antidispética, antiflatulenta, leishmanicida, cicatrizante, neuroprotetora e outras (ACEVEDO et al., 2016; NICOLETTI et al., 2015).

Muri; Sposito; Metsavaht (2009) e Azambuja (2009) esclarecem que o processo inflamatório se caracteriza como uma resposta a uma lesão tecidual gerada por algum agente mecânico, químico ou físico, no qual atua como um mecanismo fisiopatológico de defesa representado como uma cascata de reações do organismo. Essa reação inflamatória objetiva eliminar, diluir ou isolar o agente causador da lesão, agindo tanto como uma resposta defensiva quanto reparadora. Um dos fatores que podem interferir no processo inflamatório é a presença de espécies reativas de oxigênio (ERO) e/ou nitrogênio (ERN) em excesso, que representa um estresse oxidativo.

As EROs e ERNs são substâncias altamente reativas e instáveis capazes de se ligar rapidamente a outras moléculas e desempenhar papéis cruciais em diversas etapas do metabolismo normal do ser humano, podendo assumir funções que favoreçam ou prejudiquem o organismo. Quando em excesso, essas substâncias podem provocar 
o surgimento de alterações na resposta inflamatória, mutações genéticas e morte celular (BARBOSA; SOUSA; CLEMENTE, 2019; RABELO, 2016).

A C. longa possui uma classe de substâncias denominada curcuminoides, responsáveis por desempenharem as funções anti-inflamatória e antioxidante, que unidas permitem ampliar sua aplicação aos mercados de perfumaria, têxtil, medicinal, alimentício e condimentar (FILHO et al., 2000). Desta maneira, a cúrcuma representa um importante potencial econômico dentre as mais diversas espécies vegetais de interesse científico.

De maneira geral, as plantas medicinais bem como a utilização de fitofármacos alcançaram destaque na atualidade tanto em países desenvolvidos quanto em desenvolvimento atuando como medicina alternativa (PRADO et al., 2010). Sendo assim, associa-se aos profissionais de saúde o compromisso de nortear a população quanto ao uso racional desses produtos para a promoção da saúde e qualidade de vida (CAMARGO, 2013). Diante do exposto, este artigo apresentou como objetivo a realização de uma pesquisa do tipo bibliográfica pela busca por informações que dissertem sobre a atividade antioxidante e anti-inflamatória apresentada pela espécie vegetal Curcuma longa Linn.

\section{METODOLOGIA}

A presente pesquisa foi desenvolvida através de levantamento bibliográfico com abordagem qualitativa de natureza descritiva, seguindo a metodologia descrita por Augusto et al. (2013). A revisão qualitativa de natureza descritiva consiste em um tipo de revisão que sintetiza os achados de estudos qualitativos. É uma recriação da meta análises aplicado a dados qualitativos, conforme descrito por Cardoso (2019), associado ao método de pesquisa descritiva, que observa, registra, analisa, descreve e correlaciona fatos e fenômenos sem manipulá-los. Geralmente procura descobrir a frequência com que um fenômeno ocorre e sua relação com outros fatores (GARCES, 2010).

RC: 87968

Disponível em: https://www.nucleodoconhecimento.com.br/saude/curcuma-longa 
Os artigos foram selecionados nos bancos de dados DrugBank, Google acadêmico e Scielo. Os descritores de busca foram: Curcuma longa, funções biológicas, compostos bioativos, ação antioxidante, aplicação clínica da C. longa, ação antiinflamatória e neutralização de radicais livres.

Como critério qualitativo de busca, os artigos previamente selecionados foram publicados entre os anos de 2010 e 2020, na língua inglesa e portuguesa. Os métodos de seleção por leitura das publicações foram:

a) Leitura do título da obra - caso houvesse os termos dos descritores anteriormente citados, a obra era previamente selecionada, caso não atendesse aos critérios estabelecidos, a obra era descartada;

b) Leitura do resumo - Após a pré-seleção, foi realizada a leitura do resumo com o intuito de verificar se a temática investigada nesta pesquisa era comtemplada na publicação. Caso a temática não fosse contemplada, a obra era descartada;

c) Leitura integral da obra - caso a temática fosse contemplada, a leitura integral da obra era iniciada consequentemente a seleção dos dados da pesquisa foi realizada.

Os dados dos estudos incluídos foram extraídos e resumidos em uma tabela padronizada que apresentaram as seguintes informações: nome do primeiro autor, ano de publicação, país, periódico, tipo de estudo, objetivo. Sendo que as respectivas conclusões dos artigos selecionados foram extraídas e interpretadas para embasamento teórico dos resultados e discussões.

\section{RESULTADOS E DISCUSSÕES}

\subsection{Curcuma longa L.}

A Curcuma longa L. é uma espécie vegetal pertencente à mesma família do gengibre (Zingiberaceae) e que possui origem da Índia e sudeste da Ásia, entretanto, por não precisar de ambientes específicos para seu crescimento, se

RC: 87968

Disponível em: https://www.nucleodoconhecimento.com.br/saude/curcuma-longa 
desenvolve perfeitamente em zonas tropicais e subtropicais como o Brasil, cuja produção e consumo é concentrada no Centro-oeste do país e no estado de Minas Gerais (BANIK et al., 2017). A planta, também nomeada como açafrão-da-terra, cúrcuma, falso-açafrão ou açafroa, foi introduzida na culinária brasileira e na indústria têxtil (como pigmento) por volta de 1980 (SILVA; BIEGELMEYER, 2020). Atualmente o consumo também é indicado para controle da glicemia, tratamento de distúrbios inflamatórios e dermatológicos, além de apresentar atividades antimicrobiana, antioxidante, hepatoprotetora e sob o sistema nervoso central (SANTANA; MARQUES, 2020; ALONSO, 2016).

As cúrcumas são perenes podendo alcançar entre 1,20 e 1,50 m de altura, com extensas ramificações laterais, cujas folhas e flores partem dos rizomas-mãe (BERNI et al., 2014; ALMEIDA, 2012). A parte aproveitada da planta e que apresenta a cor amarela característica é o rizoma-filho o qual possui grande parcela de amido, nutrientes, óleos essenciais (cineol, felandreno) e pigmentos naturais, sendo uma parda que é descartada e outra amarela característica da espécie denominada curcumina (SOUZA et al., 2019). O rizoma pode ser consumido de forma seca (onde é triturada), fresca ralada ou em pedaços, como chá ou ainda como fitoterápico. Também passa por uma técnica de desidratação e logo em seguida é pulverizada dando origem ao pó que é comercializado em todo o país (VERMA; KUMAR, 2020; MORETES; GERON, 2019; SUETH-SANTIAGO et al., 2015).

Em relação as substâncias isoladas da cúrcuma, mais de 100 foram relatadas, contudo as principais estão discorridas na Tabela 1.

Tabela 1 - Constituintes químicos mais relatados presentes na Curcuma longa L.

\begin{tabular}{|c|c|c|c|}
\hline UT & PAÍS & ANO & CONSTITUINTES QUIIMICOS \\
\hline $\begin{array}{l}\text { LEZ; } \\
\text { VIGO }\end{array}$ & Cuba & 2014 & $\begin{array}{l}\text { Alcaloides, quinonas, cumarinas, carotenos } \\
\text { óleos essenciais, açúcares redutores, fenóis } \\
\text { taninos, flavonoides, e glicídios ou carboidratos. }\end{array}$ \\
\hline
\end{tabular}

RC: 87968

Disponível em: https://www.nucleodoconhecimento.com.br/saude/curcuma-longa 


\begin{tabular}{|l|l|l|l|}
\hline BORBA et al. & Brasil & 2016 & $\begin{array}{l}\text { Ar-tumerona, a-tumerona, curlona, a-felandreno, } \\
1,8 \text {-cineol e } \alpha \text {-zingibereno. }\end{array}$ \\
\hline RAFAEL et al. & Peru & 2018 & $\begin{array}{l}\text { Carboidratos, compostos fenólicos, taninos, } \\
\text { flavonoides, antraquinonas, triterpenos, } \\
\text { alcaloides e heterosídeos. }\end{array}$ \\
\hline SANTOS et al. & Brasil & 2019 & $\begin{array}{l}\text { Compostos fenólicos, taninos, flavonoides, } \\
\text { cumarinas, esteroides, triterpenos e açúcares } \\
\text { redutores. }\end{array}$ \\
\hline
\end{tabular}

Fonte: Autoras (2020).

Prasad; Aggarwal (2011) defendem que na raiz (parte utilizada) o constituinte presente em maior quantidade geralmente é a turmerona, um óleo volátil. Todavia, Souza et al. (2019); Ferreira (2014) discorrem que a presença ou ausência de substâncias, bem como suas quantidades presentes na planta diferem de uma região para outra devido ao tipo de solo, clima, tipo de cultivo e presença de pragas na área, da armazenagem e do transporte.

Sobre a toxicidade da C. longa, Oyemitan et al. (2017) analisaram a dosagem do óleo essencial responsável pela letalidade em camundongos do estudo. $O$ óleo causou zero e $100 \%$ de mortalidade em 2.000 e 2.900 mg/kg, por via oral, respectivamente, enquanto zero e $100 \%$ de morte foi observada em 600 e 800 $\mathrm{mg} / \mathrm{kg}$, por via intraperitoneal. Portanto, os valores de DL50 estimados para a amostra foram $2154 \mathrm{mg} / \mathrm{kg}$ (levemente tóxico), via oral, e $693 \mathrm{mg} / \mathrm{kg}$, via intraperitoneal (moderadamente tóxico). A baixa biodisponibilidade oral pode ser responsável pela ampla variação no efeito letal deste óleo em comparação com seu efeito após a administração intraperitoneal, conforme observado neste estudo. A implicação desse resultado de toxicidade aguda é que em altas doses o óleo pode ser prejudicial devido ao efeito tóxico acumulativo, portanto, seu consumo como tempero, condimento, receitas de medicamentos e agentes aromatizantes deve ser cauteloso e monitorado de perto (EUROPEAN MEDICINES AGENCY, 2010). Não há 
dados farmacocinéticos ou toxicocinéticos de estudos em humanos sobre o óleo essencial dessa planta (OYEMITAN et al., 2017).

Também é comumente relatada a possível interferência em medicamentos alopáticos quando dar-se o consumo concomitante com a cúrcuma, uma vez que esta espécie desenvolve efeitos anticoagulante, hipoglicêmico, antiestrogênico além de inibição do complexo CYP1A1, CYP1A2 e CYP3A4 e inibição da glicoproteína-P que é transportadora de medicamentos. Um exemplo de droga que tem o metabolismo diretamente interferido pela planta é o talinolol (KOZIOLEK, 2019; BAHRAMSOLTANI; RAHIMI; FARZAEI, 2017), entretanto, qualquer medicamento que tenha a metabolização realizada pelos complexos citados, está sujeito a alterações da atividade farmacológica, podendo, inclusive, agravar o quadro clínico do indivíduo que o consome.

\subsection{CURCUMINA}

A curcumina (Figura 1), também denominada diferuloilmetano é uma substância fenólica que foi isolada pioneiramente em 1815, entretanto, o primeiro artigo só foi publicado em 1937 onde abordava sobre a sua aplicação em humanos (CORREIA, 2018; FADUS et al., 2017). Esse artigo relatava sobre o uso de solução intravenosa contendo curcumina em pessoas saudáveis e seu efeito sobre o esvaziamento da vesícula biliar, sugerindo que essa substância poderia ser empregada no tratamento da colecistite subaguda, recorrente ou crônica (SUSANA, 2018; FADUS et al., 2017), sendo, portanto, considerada o princípio ativo do açafrão estando presente no rizoma da planta (ZHANG, 2013).

RC: 87968

Disponível em: https://www.nucleodoconhecimento.com.br/saude/curcuma-longa 
Figura 1 - Estrutura química da curcumina presente na Curcuma longa L.

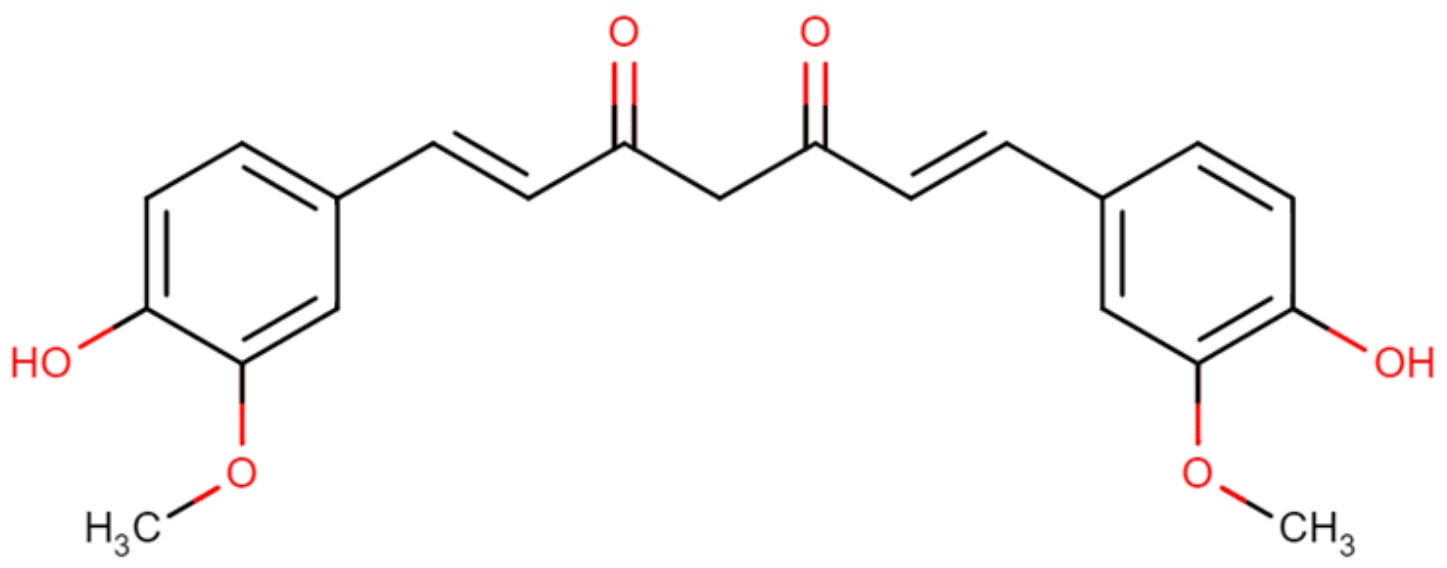

Fonte: Drugbanks (2020).

A curcumina é apontada como não nociva ao organismo mesmo quando ingerida em altas doses e, por conseguinte foi categorizada pelo Instituto Nacional de Câncer como "geralmente reconhecida como segura" (GRAS) (KUNNUMAKKARA et al., 2017; PULIDO-MORAN, 2016). Contudo, sua administração por via oral, intravenosa/intraperitoneal apresenta baixa disponibilidade que ocorre devido à baixa absorção gastrintestinal, rápida eliminação e baixa solubilidade aquosa que a curcumina apresenta, dificultando sua aplicação na prática clínica (CORREIA, 2018; STANIĆ, 2017).

Metzler et al. (2013) demonstram que, apesar da administração de doses altas de curcumina, após doses diárias de 4-8 g por 3 meses, a concentração sérica máxima observada foi de $1,3 \mu \mathrm{g} \cdot \mathrm{mL}^{-1}$ e nenhuma quantidade foi encontrada na urina. Dessa maneira, os testes em sua maioria indicam que a biodisponibilidade oral da curcumina é de apenas 1\% (LIU et al., 2016).

Surpreendentemente, a curcumina parece não ser metabolizada pelo citocromo P450, uma vez que nenhum produto de desmetilação ou hidroxilação foi detectado após a incubação da curcumina em microssomas de fígado de rato (METZLER et al., 
2013). Quando consumida por via oral, a maior parte da curcumina é excretada pelas fezes, sugerindo que apenas uma pequena porção é absorvida pelo intestino e que sofre, em seguida, rápida metabolização no fígado e plasma (LIU et al., 2016).

A curcumina sofre degradação química em soluções aquoso-orgânicas e a degradação aumenta conforme o pH torna-se básico, o que é uma grande preocupação em suas aplicações (PRIYADARSINI, 2014). Em soluções diluídas (soluções micromolares) $90 \%$ de curcumina degrada em 30 minutos (SHEN; Jl, 2012). Embora não seja totalmente compreendido, acredita-se que a degradação ocorre por reações de hidrólise através da porção diceto (PRIYADARSINI, 2014). A curcumina sofre degradação muito mais rápida quando exposta à luz solar. É uma observação comum que manchas de curcumina/cúrcuma podem ser removidas rapidamente por exposição à luz solar (SINGH, 2010).

\subsection{ATIVIDADE ANTI-INFLAMATÓRIA}

A inflamação é uma resposta homeostática em organismos contra várias condições. No entanto, quando esta resposta é excessiva, pode resultar no surgimento de diversas doenças como aterosclerose, artrite reumatoide e asma. Portanto, existem vários estudos em andamento com o objetivo de diminuir a ocorrência de inflamações excessivas (LEE et al., 2020).

Nesse sentido, análises realizadas com a cúrcuma demonstraram que ela é capaz de inibir a produção de proteínas que fazem parte do processo inflamatório no sistema nervoso, atuando na supressão das cascatas sinalizadoras inflamatórias, principalmente na diminuição da quantidade de neutrófilos circulando no período de pós-lesão, limitando, assim, os resultados negativos ocasionados por essa cascata, entretanto, o mecanismo de ação da cúrcuma não é totalmente elucidado (ROCHA, 2017, SANIVARAPU; VALLABHANENI; VERMA, 2016).

Saiz de cos (2014) enfatiza uma função importante da cúrcuma na proteção do trato grastrointestinal através do seu principal metabólito, a curcumina, que age

RC: 87968

Disponível em: https://www.nucleodoconhecimento.com.br/saude/curcuma-longa 
impedindo a ativação dos principais fatores de transcrição (fator nuclear kB (NF kB) e as B-catequinas) que desempenham um papel chave no processo inflamatório dos intestinos. Barreto (2018); Mohanty; Sahoo (2017); He et al. (2015) dissertam que o mecanismo pelo qual a cúrcuma expressa esse feito farmacológico é impedindo a produção da ciclooxigenase-2 (COX-2) além de inibir a expressão de interleucinas e fator de necrose tumoral- $\alpha$ (TNF- $\alpha$ ).

Além disso, a propriedade anti-inflamatória da $C$. longa está relacionada a sua intervenção na via do ácido araquidônico e interrupção da atividade do fator nuclear K B (NF)-KB a qual está intimamente envolvida na regulação das enzimas próinflamatórias COX-2, mieloperoxidase (MP7O), isoforma indutível da sintase do monóxido de azoto (iNOS) e 5-lipooxigenase (LOX). Na doença inflamatória intestinal (DII) a inibição de (NF)-kB parece ser o alvo principal para o tratamento da patologia e, portanto, a cúrcuma demonstra ser uma espécie promissora no que tange a remissão dos doentes além de poder ser utilizada como terapia auxiliar (OLIVEIRA et al., 2020; PROKIPCHUK, 2017).

Em relação a inflamações gengivais, Waghmare et al. (2011) desenvolveram enxaguatórios bucais a base de fitoterápicos e, em testes in vitro, evidenciaram os efeitos satisfatórios da Curcuma longa L. que apresentou cerca de $61 \%$ de eficácia na redução da inflamação gengival. Esse resultado, segundo os autores, pode ser derivado da ação inibitória da síntese de prostaglandinas apresentada pela espécie.

Ramadan; Al-Kahtani; El-Sayed (2011) analisaram o óleo essencial de C. longa frente a um edema em murinos em um modelo de inflamação aguda onde houve redução de 61,1\% da inflamação na terceira hora de administração da dose de 1000 $\mathrm{mg} / \mathrm{kg}$, e redução de $50 \%$ na dose de $500 \mathrm{mg} / \mathrm{kg}$. Dessa forma, o óleo essencial mostrou inibição dose-dependente da segunda fase do edema de pata, sugerindo a inibição de mediadores inflamatórios e eliminação de radicais livres. Também houve aumento significativo na atividade da glutationa S-transferase (GST) no fígado de todos os animais tratados com óleo de cúrcuma. Como a GST está envolvida na desintoxicação de carcinógenos, os resultados indicam que o óleo de cúrcuma

RC: 87968

Disponível em: https://www.nucleodoconhecimento.com.br/saude/curcuma-longa 
aumenta a desintoxicação de carcinógenos e pode, inclusive, reduzir a incidência de câncer.

Existem várias causas conhecidas de inflamação, e a via NF-kB/COX-2/iNOS é um conhecido importante mecanismo. Notavelmente, NF-kB controla vários estágios de inflamação e modulação imunológica por meio da regulação de várias moléculas, incluindo citocinas (por exemplo, IL-1 1 , TNF- $\alpha$ ), iNOS e quimiocinas (LEE et al., 2020). Em seus estudos, Lee et al. (2020), produziram um composto a base de uma associação de $C$. longa e $A$. Hookeri e o testaram, in vitro, na via NF-kB/COX2/iNOS e obtiveram resultados de supressão dos níveis de várias citocinas relacionadas à inflamação, incluindo IFN- $\gamma, \mathrm{IL}-1 \beta, \mathrm{IL}-6, \mathrm{IL}-13$ e IL-17 na dose de 50 $\mathrm{mg} / \mathrm{kg}$. Resultados semelhantes foram obtidos nos estudos de Kim et al. (2016) em doses menores de $0-40 \mu \mathrm{g} / \mathrm{ml}$ do extrato $\mathrm{n}-\mathrm{BuOH}$ das partes aéreas da $C$. longa, e nos estudos de Zhang et al. (2017) com óleo essencial de uma amostra coletada na China o qual apresentou inibição inflamatória de 69,22\% na dosagem de 100 mg/kg.

Já o extrato etanólico da cúrcuma e da canela, Qabaha; Abu-Lafi; Al-Rimawi (2017) demonstraram que a cúrcuma apresentou maior efeito anti-inflamatório para a inibição da secreção da IL-6 quando comparado ao efeito desenvolvido pela canela, entretanto, o melhor efeito anti-inflamatório foi observado para a TNF-a.

Em casos de esteatose hepática não-alcóolica, determinadas células, denominadas células de Kupffer, induzem a produção das citocinas inflamatórias TNF-a, IL-1b e IL-6 que podem danificar o fígado. Desta maneira, ensaios com o extrato de água quente de açafrão-da-terra demonstraram melhora no quadro de fibrose hepática induzida em camundongos, bem como atenuação da expressão de RNA mensageiro dos fatores inflamatórios (citocinas), sugerindo, portanto, que essa planta pode prevenir quadros de patologias do fígado (UCHIO; MUROSAKI; ICHIKAWA, 2018).

Já no sentido de inflamações cutâneas, a cúrcuma se mostrou eficaz (60,73\%) sob a forma de óleo essencial quando aplicada em inflamações de camundongos na concentração de 1\% utilizando acetona como veículo (KUMAR et al., 2018), sendo

RC: 87968

Disponível em: https://www.nucleodoconhecimento.com.br/saude/curcuma-longa 
dose-dependente. Essa ação anti-inflamatória também culminou na redução do peso da orelha dos animais (local da inflamação induzida) bem como redução das citocinas pró-inflamatórias. Entretanto, os autores evidenciam a importância da realização de mais testes diferentes para esse mesmo extrato afim de se obter maiores informações acerca do mecanismo de ação da espécie, uma vez que não há dados científicos suficiente publicados.

Diante das falhas no tratamento do câncer, novas terapias com produtos naturais como o açafrão têm sido consideradas. Dessa maneira, ao avaliar o efeito da cúrcuma in vitro em queratinócitos, fibroblastos e em um carcinoma epidermoide oral, Campos et al. (2017) constataram que nas doses mais altas a cúrcuma promove a morte celular de células normais e cancerígenas, desenvolvendo, portanto, um potencial citotóxico, enquanto doses mais baixas do fármaco mostraram efeito significativo na redução da migração celular e adesão célula-célula. Em contrapartida, a administração local de $C$. longa em um modelo de xenoenxerto canceroso inibiu o crescimento do tumor e microscopicamente, foi possível associar esse resultado a um fenótipo histológico mais diferenciado e menos agressivo. Portanto, a cúrcuma é um composto natural muito promissor na busca pela estagnação ou regressão do câncer bucal, mas o estudo do mecanismo de ação desse medicamento ainda é necessário.

\subsection{ATIVIDADE ANTIOXIDANTE}

Os antioxidantes podem ser definidos como compostos que inibem ou retardam a oxidação de outras moléculas ao inibir a iniciação ou propagação de reações em cadeia de oxidação. Os antioxidantes protegem o corpo humano dos radicais livres e dos efeitos das EROS (Espécies Reativas de Oxigênio), desacelerando o progresso de muitas doenças crônicas, bem como a peroxidação lipídica. Além disso, eles têm sido amplamente utilizados como aditivos alimentares para fornecer proteção contra a degradação oxidativa dos alimentos (MIAH et al., 2018). 
Ao tratar sobre o aspecto antioxidante da C. longa L., Singh et al. (2010) elucidam que o óleo essencial dessa espécie apresenta atividade antioxidante devido a sinergia dos seus componentes. O estresse oxidativo é comumente relacionado a circunstâncias de ameaça de vida como doenças neurodegenerativas, câncer, diabetes mellitus e doenças cardiovasculares (KAUR; CHAUHAN; SANDHIR, 2011; SUN; ONG; NYAM, 2017). Dessa maneira, existe a hipótese de que há uma conexão entre as substâncias bioativas e a ação antioxidante da planta (YANG et al., 2020).

Avanço et al. (2017) encontraram resultados de potencial antioxidante dosedependente do óleo essencial de $C$. longa L., sugerindo que o óleo expressa sua ação antioxidante através da doação de hidrogênio. A possível diferença de resultados que se possam encontrar para esse mesmo ensaio pode ser devido ao baixo percentual de ar-tumerona, uma vez que quando esta é reduzida, demonstra diminuição da capacidade de eliminação das espécies reativas (ou radicais livres) presentes no meio.

Os extratos obtidos por extração ultrassônica manifestaram maior capacidade redutora e capacidade superior de eliminação dos radicais 2,2-difenil-1-picril-hidrazil (DPPH) e [2,2'-azino-bis(3-ethylbenzothiazoline)-6-sulfonic acid] (ABTS) quando comparado aos extratos com solventes convencionais. Além disso, os resultados também demonstraram que in vitro a C. longa L. apresenta potencial anticâncer considerável ao reduzir a produção de espécies reativas de oxigênio (EROs) intracelular, inibição da migração celular, indução de alterações morfológica nas células e em material genético. Entretanto, ainda são necessários estudos mais aprofundados in vivo para compreender questões fisiológicas relacionadas, como a biodisponibilidade e o metabolismo desses extratos (YANG et al., 2020).

Quando se submeteu a C. longa L. a extração com água, álcool metílico e n-hexano, os resultados alcançados demonstram que todos apresentam atividade antioxidante, entretanto, os resultados do extrato com álcool metílico foram relativamente superiores, sugerindo que esse resultado se deve a presença de flavonoides, 
glicosídeos cardíacos, taninos e compostos fenólicos presentes no extrato de maior polaridade (MOHAMMED, 2020). O autor também analisou os possíveis resultados in vivo que a planta apresentaria e encontrou que a administração das frações do extrato metílico da Curcuma longa L. por 21 dias resultou em uma redução acentuada da hiperglicemia em ratos diabéticos.

A desordem no equilíbrio oxidante/antioxidante em favor do primeiro acarreta uma situação de estresse oxidativo, que é sabidamente um componente dos mecanismos de dano ao tecido molecular e celular em um amplo espectro de doenças humanas que leva à patogênese das complicações diabéticas (MOHAMMED et al., 2017). Embora estudos tenham demonstrado que o mecanismo responsável pela diminuição da atividade dessas enzimas no estado diabético não seja totalmente conhecido, os resultados obtidos podem ser decorrentes da fosforilação e diminuição da expressão dessas enzimas (SWETA; SEEMI, 2012).

Em um estudo clínico, duplo-cego randomizado e controlado por placebo, ao utilizar a cúrcuma para o tratamento de osteoartrite de joelho, além da melhora clínica, houve diminuição dos biomarcadores relacionados à doença; o biomarcador de inflamação (IL-1b) e de estresse oxidativo (EROS e MDA) apresentaram redução significativa. A melhora clínica na Escala Visual Analógica e no Índice de Osteoartrite das Universidades de Western Ontario e McMaster pode ser subjetiva, mas os níveis dos biomarcadores são certos parâmetros que retratam o status da atividade anti-inflamatória e do estresse oxidativo (antioxidante). Foi estabelecido que há um aumento desses marcadores em várias doenças e a redução desses marcadores está fortemente correlacionada com o nível da doença (SRIVASTAVA et al., 2016).

Os compostos presentes na cúrcuma, também desenvolvem ação antioxidante ao diminuir a peroxidação lipídica por meio da conservação da atividade das enzimas antioxidantes superóxido dismutase, catalase e glutationa peroxidase em níveis elevados. As propriedades antioxidantes dos extratos de açafrão são devidas principalmente à diacetilcurcumina, desmetoxicurcumina e bisdemetoxicurcumina 
(SANTOS-SÁNCHEZ et al., 2017). Entretanto, Braga et al. (2018) elucidam que diferenças no processo de secagem das folhas de cúrcuma parecem estar diretamente relacionadas à degradação de componentes de ação antioxidante (como os compostos fenólicos), evidenciada pela mudança de cor das folhas quando secadas a altas temperaturas ou longo tempo de secagem.

\section{CONCLUSÃO}

Foi possível elucidar que a Curcuma longa L. apresenta capacidade anti-inflamatória e antioxidante comprovada in vitro, porém, não há publicações em humanos que comprovem as ações farmacológicas. Também é possível comprovar a escassez de estudos brasileiros. Em relação a atividade anti-inflamatória, os resultados demonstraram que a cúrcuma age diretamente em substâncias essenciais para a promoção da inflamação, como o bloqueio da produção da ciclooxigenase-2 (COX2), inibição da expressão de interleucinas e fator de necrose tumoral- $\alpha$ (TNF- $\alpha$ ), além de interromper a atividade do fator nuclear kB (NF)-kB. Quanto aos estudos que abordaram ensaios antioxidantes, a planta apresentou alta capacidade redutora e/ou eliminadora de substâncias oxidantes que podem danificar o organismo, sugerindo, inclusive, uma espécie que tem potencial anticâncer.

\section{REFERÊNCIAS}

ACEVEDO, Jorge Luis Arroyo; QUINO, César Ivanovish Franco; FIGUEROA, Yovani Martin Condorhuamán; CORAL, José Gonzalo Cabanillas; ASMAT, Roberto Jesús Chávez; GUZMAN, Andrea Anampa. Toxicidad a 90 días del extracto atomizado de rizoma de Curcuma longa (A4R), flores de Cordia lutea (A4F) y hojas de Annona muricata (A4I) en modelo murino. Rev. Peru Med Integ. v. 1, n. 4, p. 5-10, 2016.

ALMEIDA, Mariana Corrêa. Efeitos do processamento por radiação em espécies da família zingiberaceae: açafrão (Curcuma longa L.), gengibre (Zingiber officinale Roscoe) e zedoária (Curcuma zedoaria (Christm.) Roscoe). 
Dissertação (Tecnologia Nuclear). Instituto de Pesquisas Energéticas e Nucleares IPEN, São Paulo - SP. 2012.

ALONSO, J. R. Curcuma. In: Tratado de Fitofarmácos e Nutracêuticos. São Paulo: A C Farmacêutica, p. 364 - 373. 2016.

AUGUSTO, Cleiciele Albuquerque; SOUZA, José Paulo de; DELLAGNELO, Eloise Helena Livramento; CARIO, Silvio Antonio Ferraz. Pesquisa Qualitativa: rigor metodológico no tratamento da teoria dos custos de transação em artigos apresentados nos congressos da Sober (2007-2011). Revista de Economia e Sociologia Rural, v. 51, n. 4, p. 745-764, 2013.

AVANÇO, Geleys Avanço; FERREIRA, Flavio Dias; BOMFIM, Natália Silva; SANTOS, Paula Andréia de Souza Rodrigues dos; PERALTA, Rosane Marina; BRUGNARI, Tatiane; MALLMANN, Carlos Augusto; FILHO, Benício Alves de Abreu; MIKCHA, Jane Martha Graton; JR, Miguel Machinski. Curcuma longa L. essential oil composition, antioxidant effect, and effect on Fusarium verticillioides and fumonisin production. Food Control, v. 73, p. 806-813, 2017.

AZAMBUJA, Marcos Schuch de. Ação antiinflamatória, imunomoduladora e citotóxica do composto RDV 8. Dissertação de Mestrado. Pontifícia Universidade Católica do Rio Grande do Sul. 2009.

BAHRAMSOLTANI, Roodabeh; RAHIMI, Roja; FARZAEI, Mohammad Hosein. Interações farmacocinéticas de curcuminóides com drogas convencionais: uma revisão J. Ethnopharmacol. v. 209, p. 1 - 12, 2017.

BANIK, Urmila; PARASURAMAN, Subramani; ADHIKARY, Adhikary Kumar; ORTHMAN, Nem Hayati. Curcumin: the spicy modulator of breast carcinogenesis. Jour Exp Clin Cancer Res.v. 36, n. 1, 2017.

BARBOSA, Dafne Nayara Quinta; SOUSA, Haylla Freiria Maganhoto de; CLEMENTE, Rodolfo Castilho. Ação antioxidante dos alimentos. DESAFIOS-

RC: 87968

Disponível em: https://www.nucleodoconhecimento.com.br/saude/curcuma-longa 
Revista Interdisciplinar da Universidade Federal do Tocantins, v. 6, n. 4, p. 1-2, 2019.

BARRETO, Catarina Sofia Vitorino. O contributo da Fitoterapia na Cicatrização de Feridas. Tese de Doutorado. Universidade de Coimbra. 2018.

BERNI, R. F.; CHAVES, F. C. M.; PINHEIRO, J. B.; VAZ, A. P. A. Produção de açafrão em função de acessos e do peso de rizomas-semente. Revista Brasileira de Plantas Medicinais, Campinas, v. 16, n. 3, p. 765-770, 2014.

BRAGA, Monick Cristina; VIEIRA, Ellen Caroline Silverio; CALIARI, Márcio; OLIVEIRA, Tatianne Ferreira de. Curcuma longa L. Leaf: Kinetic Study Using Different Drying Methods. Adv Food Process Technol: AFPT-110, v. 10, 2018.

BRASIL, Ministério da Saúde. Secretaria de Ciência, Tecnologia e Insumos Estratégicos. Departamento de Assistência Farmacêutica. Política e Programa Nacional de Plantas Medicinais e Fitoterápicos Brasília: Ministério da Saúde, 2016.

CAMARGO, Sula de; PEREIRA, Vera Barros Leça. A prática da Fitoterapia pelo Nutricionista -algumas reflexões. RASBRAN. São Paulo. Ano 5, n. 1, 2013.

CAMPOS, Paloma Santos de; MATTE, Bibiana Franzen; DIEL, Leonardo Francisco; JESUS, Luciano Henrique; BERNARDI, Lisiane; ALVEES, Alessandro Menna; RADOS, Pantelis Varvaki; LAMERS, Marcelo Lazzaron. Low doses of curcuma longa modulates cell migration and cell-cell adhesion. Phytotherapy Research, v. 31, n. 9, p. 1433-1440, 2017.

CARDOSO, Vanessa; TREVISAN, Ivana; CICOLELLA, Dayane de Aguiar; WATERKEMPER, Roberta. Revisão sistemática de métodos mistos: método de pesquisa para a incorporação de evidências na enfermagem. Texto \& ContextoEnfermagem, v. 28, 2019. 
CORREIA, Soraia Filipa Santos Pina. Curcumina-A sua aplicabilidade na terapêutica da Diabetes Mellitus. Tese de Doutorado. Universidade de Coimbra. 2018.

DRUGBANKS. Curcumina structure. Acesso em: set. de 2020 <https://www.drugbank.ca/structures/DB11672/image.svg>

EUROPEAN MEDICINES AGENCY. Report on Curcuma longa L. Rhizoma. Committee on Herbal Medicinal Products (HMPC) Assessment, European Medicines Agency: Evaluation of Medicines for Human Use, 7 Westferry Circus, London. 2010.

FADUS, Matthew C.; LAU, Cecilia; BIKHCHANDANI, Jai; LYNCH, Henry T. Curcumin: An age-old antiinflammatory and anti-neoplasic agent. Journal of Traditional and complementary Medicine. 2017.

FERREIRA, P. P. Extração, caracterização e aplicação de fécula de açafrão (Curcuma longa L.) no desenvolvimento de biscoito. Dissertação. Goiás: Universidade Federal de Goiás/UFG, 2014.

FILHO, Arthur Bernardes Cecilio Filho; SOUZA, Rovilson José de; BRAZ, Leila Trevizan; TAVARES, Marcelo. Cúrcuma: planta medicinal, condimentar e de outros usos potenciais. Ciência Rural, v. 30, n. 1, p. 171-177, 2000.

GARCES, Solange Beatriz Billig. Classificação e tipos de pesquisas. Universidade de Cruz Alta-Unicruz, 2010.

GRASSO, Eliane da Costa; AOYAMA, Elisa Mitsuko; FURLAN, Marcos Roberto. Ação antiinflamatória de Curcuma longal.(Zingiberaceae). Rev. EletrThesis, v. 28, p. 122-123, 2017.

RC: 87968

Disponível em: https://www.nucleodoconhecimento.com.br/saude/curcuma-longa 
HE, Yan; YUE, Yuan; ZHENG, Xi; ZHANG, Kun; CHEN, Shaohua; DU, Zhiyn. Curcumin, inflammation, and chronic diseases: how are they linked? Molecules, v. 20, n. 5, p. 9183-9213, 2015.

KAUR, Harpreet; CHAUHAN, Shaveta; SANDHIR, Rajat. Protective effect of lycopene on oxidative stress and cognitive decline in rotenone induced model of Parkinson's disease. Neurochem. Res. v. 36, p. 1435-1443, 2011.

KIM, Dae Wook; LEE, Seon Min; WOO, Hyun Sim; PARK, Ji-Young; KO, Byoung Seob; HEO, Jeong Doo; RYU, Jovem Bae; LEE, Woo Song. Chemical constituents and anti-inflammatory activity of the aerial parts of Curcuma longa. Journal of Functional Foods, v. 26, p. 485-493, 2016.

KOZIOLEK, Mirko; ALCARO, Stefano; AUGUSTIJNS, Patrick; BASIT, Abdul W.; GRIMM, Michael; HENS, Bart; HOAD, Caroline L.; JEDAMZIK, Philipp; MADLA, Christine M.; MALIEPAARD, Marc; MARCIANI, Luca; MARUCA, Annalisa; PARROTT, Neil; PÁVEK, Petr; PORTER, Christopher J. H.; REPPAS, Christos; RIET-NALES, Diana Van; RUBBENS, Jari; CORSETTI, Maura. The mechanisms of pharmacokinetic food-drug interactions-A perspective from the UNGAP group. European Journal of Pharmaceutical Sciences, v. 134, p. 31-59, 2019.

KUMAR, Anant; AGARWAL, Karishma; SINGH, Monika; SAXENA, Archana; YADAV, Pankaj; MAURYA, Anil Kumar; YADAV, Anju; TANDON, Sudeep; CHANDA, Debabrata; BAWANKULE, Dnyaneshwar U. Essential oil from waste leaves of Curcuma longa L. alleviates skin inflammation. Inflammopharmacology, v. 26, n. 5, p. $1245-1255,2018$.

KUNNUMAKKARA, Aajaikumar B.; BORDOLOI, Devivasha; PADMAVATHI, Ganesan; MONISHA, Javadi; ROY, Nand Kishor; PRASAD, Sahdeo; AGGARWAL, Bharat B. Curcumin, the golden nutraceutical: multitargeting for multiple chronic diseases. British Journal of Pharmacology. 2017.

RC: 87968

Disponível em: https://www.nucleodoconhecimento.com.br/saude/curcuma-longa 
LEE, Soon-Young; CHO, Seung-Sik; LI, YongChun; BAE, Chun-Sik; MOK, Parque Kyung; DAE-HUN, Parque. Anti-inflammatory Effect of Curcuma longa and Allium hookeri Co-treatment via NF-kB and COX-2 Pathways. Scientific reports, v. 10, n. 1, p. 1-11, 2020.

LIU, Weidong; ZHAI, Yingjie; HENG, Xueyuan; CHE Yuan Feng; CHEN, Wenjun; SUN, Dezhong; ZHAI, Guangxi. Oral bioavailability of curcumin: problems and advancements. Journal of drug targeting, v. 24, n. 8, p. 694-702, 2016.

MARCHI, Juliana Pelissari; TEDESCO, Luana; MELO, Ailton da Cruz; FRASSON, Andressa Caroline; FRANÇA, Vivian Francielle; SATO, Samantha Wietzikoski; LOVATO, Evellyn Claudia Wietzikoski. Curcuma longa L., o açafrão da terra, e seus benefícios medicinais. Arquivos de Ciências da Saúde da UNIPAR, v. 20, n. 3, 2016.

MIAH, Tuhin; SULTANA, Jabin; UDDIN, Md. Forhad; JALAL, Shah; SINGHA, Shuvo; DEY, Tuli. PODDAR, Sonnet. In-vitro antioxidant properties of Ocimum tenuiflorum, Curcuma longa and Camellia sinensis. International Journal of Natural and Social Sciences. v. 4, n. 1, p. 78-83, 2018.

MORETES, Débora Nogueira; GERON, Vera Lúcia Matias Gomes. Os benefícios medicinais da Curcuma longa L.(açafrão da terra). Revista Científica da Faculdade de Educação e Meio Ambiente, v. 10, n. 1, p. 106-114, 2019.

MURI, Estela Maris Freitas; SPOSITO, Maria Matilde de Mello; METSAVAHT, Leonardo. Antiinflamatórios não-esteroidais e sua farmacologia local. Acta fisiátrica, v. 16, n. 4 , p. 186-190, 2009.

METZLER, Manfred; PFEIFFER, Erika; SCHULZ, Simone I; DEMPE, Julia S. Curcumin uptake and metabolism. Biofactors, v. 39, n. 1, p. 14-20, 2013.

RC: 87968

Disponível em: https://www.nucleodoconhecimento.com.br/saude/curcuma-longa 
MOHAMMED, A.; WUDIL, A. M.; ALHASSAN, A. J.; IMAM, A. A.; MUHAMMAD, I. U.; IDI, A. Hypoglycemic activity of Curcuma longa Linn root extracts on alloxan induced diabetic rats. Saudi Journal of Life Science. v. 2, p. 43-49, 2017.

MOHAMMED, Abdulrashid; USMAN, Muhammad Ibrahim; WUDIL, Alhassan Muhammad; ALHASSAN, Adamu Jibrin; ABUBAKAR. Salisu Maiwada; LAT, Ngwen Anthony. In vitro and in vivo Antioxidant Properties of Extracts from the Root of Curcuma longa Linn. European Journal of Medicinal Plants, p. 6-12, 2020.

MOHANTY, Chandana, SAHOO, Sanjeeb K. Curcumin and its topical formulations for wound healing applications. Drug Discovery Today. v. 22, n. 10, p. 1582-1592, 2017.

NICOLETTI, Maria Aparecida; ITO, Rosilene Kinue; FUKUSHIMA, André Rinaldi; LEANDRO, Aline Camata. Farmacovigilância de drogas vegetais e seus derivados: uma ação necessária e já iniciada para a segurança do paciente, no contexto do uso racional de medicamentos. Vig Sanit Debate. 2015.

OLIVEIRA, Carla Braga Campelo de; BRITO, Lorena Almeida; FREITAS, Morgana Andrade; SOUZA, Marcos Porto Arrais de; RÊGO, Juliana Magalhães da Cunha; MACHADO, Richele Janaína de Araújo. Obesidade: inflamação e compostos bioativos. Journal of Health \& Biological Sciences, v. 8, n. 1, p. 1-5, 2020.

OYEMITAN, Idris A.; ELUSIYAN, Christianah A.; ONIFADE, Ayoola O.; AKANMU, Moses A.; OYEDEJI, Adebola O.; McDONALD, Armando G. Neuropharmacological profile and chemical analysis of fresh rhizome essential oil of Curcuma longa (turmeric) cultivated in Southwest Nigeria. Toxicology reports, v. 4, p. 391-398, 2017.

PRADO, Caroline Nunes do; NEVES, Dayse Regina de Jesus; SOUZA, Helena Dias de; NAVARRO, Francisco. O uso de fitoterápicos no tratamento da obesidade. Rev. Bras. Obes. Nutr. Emagrec. São Paulo. v. 4, 2010.

RC: 87968

Disponível em: https://www.nucleodoconhecimento.com.br/saude/curcuma-longa 
PRASAD, S. E; AGGARWAL, B. B. Açafrão, o tempero dourado: da medicina tradicional à medicina moderna. In Herbal Medicine: Biomolecular and Clinical Aspect: Second Edition, p. 263-288, 2011.

PROKIPCHUK, Anna. Fitoterapia na doença inflamatória intestinal. Tese de Doutorado. 2017.

PRIYADARSINI, Kavirayani Indira. The chemistry of curcumin: from extraction to therapeutic agent. Molecules, v. 19, n. 12, p. 20091-20112, 2014.

PULIDO-MORAN, Mario; MORENO-FERNANDEZ, Jorge; . Curcumin and Health. Molecules Journal. 2016.

QABAHA, Khaled; ABU-LAFI, Saleh; AL-RIMAWI, Fuad. Anti-inflammatory Activities of Ethanolic Extracts of Curcuma Longa (Turmeric) and cinnamon (Cinnamomum verum). 2017.

RABELO, Thallita Kelly. Propriedades antioxidantes, anti-inflamatórias e antinociceptivas do ácido chiquímico. 2016.

RAMADAN, Gamal; AL-KAHTANI, Mohammed Ali; EL-SAYED, Wael Mohamed. Anti-inflammatory and anti-oxidant properties of Curcuma longa (turmeric) versus Zingiber officinale (ginger) rhizomes in rat adjuvant-induced arthritis. Inflammation, v. 34, n. 4, p. 291-301, 2011.

ROCHA, Valéria Mendes da. Efeitos da cúrcuma na recuperação funcional após hemissecção medular. Tese de Doutorado. Universidade de São Paulo. 2017.

SAIZ DE COS, Paula. Cúrcuma I (Curcuma longa L.). Reduca (Biología). Serie Botánica. 2014.

RC: 87968

Disponível em: https://www.nucleodoconhecimento.com.br/saude/curcuma-longa 
SANIVARAPU, Raghavendra; VALLABHANENI, Vijayalakshmi; VERMA, Vivek. The Potential of Curcumin in Treatment of Spinal Cord Injury. Neurology Research International, v. 2016, 2016.

SANTANA, Cícero Márcio Cruz; MARQUES, Ana Emilia Formiga. Atividades farmacológicas do extrato da Curcuma longa L.: uma revisão de literatura. Revista Ciência (In) Cena, v. 1, n. 10, p. 18-28, 2020.

SANTOS-SÁNCHEZ, Norma F.; SALAS-CORONADO, Raúl; VALADEZ-BLANCO, Rogelio; HERNÁNDEZ-CARLOS, Beatriz; GUADARRAMA-MENDOZA, Paula C. Natural antioxidant extracts as food preservatives. Acta Scientiarum Polonorum Technologia Alimentaria, v. 16, n. 4, p. 361-370, 2017.

SHEN, Liang; Jl, Hong-Fang. The pharmacology of curcumin: is it the degradation products? Trends Mol. Med. v. 18, p. 138-143, 2012.

SILVA, Ayla Winnie Ramos da; BIEGELMEYER, Renata. Curcuma longa L. (Zingiberaceae): desenvolvimento tecnológico para aplicação como agente terapêutico auxiliar no tratamento de pacientes portadores da Doença de Alzheimer. Revista Fitos, v. 14, n. 2, p. 249-258, 2020.

SINGH, Umang; VERMA, Sandeep; GHOSH, H. N.; RATH, M. C.; PRIYADARSINI, A.; SHARMA, K. K.; PUSHPA, S. K.; SARKAR, T. Mukherjee. Photo-degradation of curcumin in the presence of $\mathrm{TiO}_{2}$ nanoparticules: Fundamentals and application. $\mathbf{J}$. Mol. Catal. A, v. 318, 106-111, 2010.

SINGH, G; KAPOOR, I. P. S.; SINGH, Pratibha; HELUANI, Carola S. de; LAMPASONA, Marina P. de; CATALAN, Cesar A. N. Comparative study of chemical composition and antioxidant activity of fresh and dry rhizomes of turmeric (Curcuma longa Linn.). Food and Chemical Toxicology, v. 48, p. 1026-1031, 2010.

RC: 87968

Disponível em: https://www.nucleodoconhecimento.com.br/saude/curcuma-longa 
SOUZA, Carolina Melo de. Efeito neuroprotetor da curcumina sobre o estresse oxidativo, inflamação, memória e dano neuronal de ratos submetidos à isquemia cerebral transitória. Tese. Universidade Federal do Ceará, 2012.

SOUZA, Sidnei Azevedo. Produtividade agroeconomica e bromatologia das plantas de açafrão da terra em função do cultivo com diferentes números de fileiras e de amontoas. 2019.

SRIVASTAVA, Shobhit Srivastava; SAKSENA, Anil K.; KHATTRI, Sanjay; KUMAR, Santosh; DAGUR, Raghubendra Singh. Curcuma longa extract reduces inflammatory and oxidative stress biomarkers in osteoarthritis of knee: a four-month, double-blind, randomized, placebo-controlled trial. Inflammopharmacology, v. 24, n. 6, p. 377388, 2016.

SUETH-SANTIAGO, Vitor; MENDES-SILVA, Gustavo Peron; DECOTÉ-RICARDO, Débora; LIMA, Marco Edilson Freire de. et al. Curcumina, o pó dourado do açafrãoda-terra: Introspecções sobre química e atividades biológicas. QuimNova. São Paulo, v. 38, n.4, p. 538-552, 2015.

SUN, Yan Yi; ONG, Wei Ting Jess; NYAM, Kar Lin. Effect of various solvents on the pulsed ultrasonis assisted extraction of phenolic compounds from Hibiscus cannabinus L. leaves. Ind. Crop. Prod. v. 140, p. 111708, 2019.

STANIĆ, Zorka. Curcumin, a compound from natural sources, a true scientific challenge-a review. Plant Foods for Human Nutrition, v. 72, n. 1, p. 1-12, 2017.

SUSANA, Marta Caldeira. Curcumina: propriedades biológicas e aplicações terapêuticas. 2018. Tese de Doutorado.

SWETA, Shailey:; SEEMI, Farhat Basir. Strengthening of antioxidant defence by Azadirachtaindicain alloxan diabetic rat's tissues. Journal of Ayurveda Integrative Medicine. v. 3, p. 130-135, 2012. 
UCHIO, Ryusei; MUROSAKI, Shinji; ICHIKAWA, Hiroshi. Hot water extract of turmeric (Curcuma longa) prevents non-alcoholic steatohepatitis in mice by inhibiting hepatic oxidative stress and inflammation. Journal of nutritional science, v. 7, 2018.

VERMA, Suruchi; KUMAR, Vikas. Pharmacological profile of turmeric oil: a review. Neuropharmacology Research Laboratory, Department of Pharmaceutics, Indian Institute of Technology (Banaras Hindu University), India. LEK SIROV. Belgrade. v. 35, n. 35, p. 3-21, 2015.

WAGHMARE, P.; CHAUDHARI, A.; KARHADKAR, V.; JAMKHANDE, A. Comparative evaluation of turmeric and chlorhexidine fluconate mouthwash in prevetion of plaque formation and gingivitis: a clinical and microbiological study. $\mathbf{J}$ Contemp Dent Pract, v. 12, n. 4, p. 221-224, 2011.

YANG, Qiong-Qiong; CHENG, Li-Zeng; ZHANG, Tongze; YARON, Sima; JIANG, Hai-Xia; SUI, Zhong-Quan; CORKE, Harold. Phenolic profiles, antioxidant, and antiproliferative activities of turmeric (Curcuma longa). Industrial Crops and Products, v. 152, p. 112561, 2020.

ZHANG Dong-wei; FU, Min; GAO, Si-Hua; LIU, Jun-Li. Curcumin and Diabetes: A Systematic Review. Evidence-based Complementary and Alternative, v. 2013, 2013.

ZHANG, Lanye; YANG, Zhiwen; CHEN, Feng; SU, Ping; CHEN, Dingkang; PAN, Wanyi; FANG, Yanxiong; DONG, Changzhi; ZHENG, Xi; DU, Zhiyun. Composition and bioactivity assessment of essential oils of Curcuma longa L. collected in China. Industrial Crops and Products, v. 109, p. 60-73, 2017.

Enviado: Maio, 2021.

Aprovado: Junho, 2021.

RC: 87968

Disponível em: https://www.nucleodoconhecimento.com.br/saude/curcuma-longa 